Supporting Information for

\title{
Selective Dispersion of Highly Pure Large-Diameter Semiconducting Carbon Nanotubes by a Flavin for Thin Film Transistors
}

\author{
Minsuk Park, ${ }^{1,+\dot{t}}$ Somin Kim, ${ }^{1,+\dot{t}}$ Hyeokjae Kwon, ${ }^{2,+\dot{t}}$ Sukhyun Hong, ${ }^{1}$ Seongil Im, ${ }^{2}$ \\ and Sang-Yong $\mathrm{Ju}^{1, *}$ \\ ${ }^{1}$ Department of Chemistry and ${ }^{2}$ Department of Physics, Yonsei University, Seoul 03722 , \\ Republic of Korea \\ * E-mail address: syju@yonsei.ac.kr \\ $\$$ Equally contributed to this work
}




\section{RBM analysis of enriched samples}

After careful calibration with respect to Si peak at $520.89 \mathrm{~cm}^{-1}$, Raman RBM frequency $\left(\omega_{\text {RBM }}\right)$ analysis on separated PSWNT and HiPco samples were conducted. Assuming the $d$ distribution as Gaussian profile, one can expect that peak intensity of resonating SWNTs would decrease when deviations from the average $d$ occurs. PSWNT has a median $d$ at $1.35 \mathrm{~nm}$ with \pm $0.4 \mathrm{~nm}$ distribution. With $785 \mathrm{~nm}$ excitation, the observed $\omega_{\mathrm{RBM}}$ peak from the supernatant of FC12-PSWNT are 161.5, 190.5, 205.6, 216.4, and $225.0 \mathrm{~cm}^{-1}$. Using the empirical diameter$\omega_{\mathrm{RBM}}$ relationship (i.e., semiconducting: $\omega_{\mathrm{RBM}}=223 / d+10$, metallic: $\omega_{\mathrm{RBM}}=218 / d+17$ ), ${ }^{\mathrm{S}-2}$ one can assign m- and s-PSWNTs for a given PSWNT. The 161.5 and $190.5 \mathrm{~cm}^{-1}$ peaks derive from m-SWNTs whereas the 205.6, 216.4 and $225.0 \mathrm{~cm}^{-1}$ peaks originate from s-SWNTs. By comparison of underlying area, s- and m-enrichments of PSWNT are determined to be 95 and $78 \%$, respectively. However, it needs to be pointed out that resonating m-SWNTs are situated at near median diameter $\left(1.35 \mathrm{~nm}\right.$ or $\left.176 \mathrm{~cm}^{-1}\right)$ and, therefore, appear to have much greater population than those of s-SWNTs, leading to overestimation of the metallic content. Similarly, HiPco has $d$ distribution ranging from 0.65 to $1.35 \mathrm{~nm}$, centered at $1.0 \mathrm{~nm} .^{3}$ With $632.8 \mathrm{~nm}$ laser (or $1.96 \mathrm{eV}$ ), observed RBM peaks $\left(\omega_{\mathrm{RBM}}\right)$ from supernatant are 195.1, 218.5, 256.3, and 285.1 $\mathrm{cm}^{-1}$. Among those peaks, the 195.1 and $218.5 \mathrm{~cm}^{-1}$ peaks originate from $\mathrm{m}-\mathrm{SWNTs}$, and the 256.3 and $285.1 \mathrm{~cm}^{-1}$ peaks derive from s-SWNTs. Because the observed s- and m-SWNTs are distributed equally from the median $d$ line, one can assume that each electronic structure would be excited with similar probability using resonating laser windows (i.e., $\pm 0.1 \mathrm{eV}$ ). By calculating each s- and m-area of respective supernatant and precipitate, the number-averaged s- and mSWNT enrichments of HiPco are determined to be 76 and $61 \%$, respectively. 


\section{Purity analysis based on absorption spectrum}

Absorption spectra containing distinct $\mathrm{S}_{22}$ and $\mathrm{M}_{11}$ peak regions were used to determine purity

of enriched s-PSWNTs, according to previously reported literature. ${ }^{\text {S4-8 }}$ SDS-dispersed PSWNT was utilized as a reference having 1 part of $\mathrm{m}$ - and 2 part of s-SWNTs. In the case of SDSPSWNTs dispersion, the summated peak areas of background-subtracted m- $(570-750 \mathrm{~nm})$ and s(750-1200 nm) absorption region are 1 and 8.74, respectively, which are tabulated in Table S2. s/m- peak ratio of FC12-PSWNTs with varying centrifugal speed in Figure 3 are 250.8, 349.5, 1152 , and 1218 for $5,10,20$, and $30 \mathrm{~kg}$ samples. Those s-/m- peak area ratio was divided by SDS-based normalization factor (i.e., 8.74) to produce s-/m- abundance ratio and s-PSWNT purity. The results are summarized in Table S2. Moreover, we added absorption spectra of a commercially available 99\% enriched s-SWNT with similar diameter, as shown in Figure S7.

\section{Raman mapping analysis}

Unfortunately, the broad $d$ distribution of PSWNT hampers us to estimate s- and m-purity based on $\mathrm{G}^{+}$and $\mathrm{G}^{-}$band intensities proposed by Finnie et al. ${ }^{\mathrm{S} 9}$ Instead, in order to verify a spatial homogeneity of purity of the deposited s-PSWNT on a Si substrate, Raman mapping probed by $532 \mathrm{~nm}$ was acquired. This measurement allows us to probe smaller $d \mathrm{~m}$ - and larger $d$ s-PSWNTs originating from $\mathrm{M}_{11}$ and $\mathrm{S}_{33}$ transitions, complementary to $d$ information obtained by $785 \mathrm{~nm}$ excitation. Figure S8 displays Raman data extracted from Raman mapping whose size is $50 \times 50 \mu \mathrm{m}^{2}$ with pixel size of $1 \times 1 \mu^{2}$. Overlaid $2500 \mathrm{RBM}$ spectra displays no particular RBM signal originating from m-PSWNT, as indicated by dotted line based on the above $d$ - $\omega_{\mathrm{RBM}}$ relationship. In addition, $\mathrm{G}$ band spectra exhibit absent BWF shape originating from m-PSWNT. In addition, summated RBM intensity shows that RBM intensity from s-PSWNTs is dominant as 
compared to that from m-PSWNTs. It is noteworthy that majority of the m-PSWNT signal originates from residual peak tails from s-PSWNT. This suggests that the enriched sample possesses not only high purity, but also have spatial electronic homogeneity. Intensity map from $\mathrm{G}^{+}$and $\mathrm{G}^{-}$bands displays that there is no deviation in the intensity pattern, suggesting the absence of BWF peak originating from m-PSWNTs. 

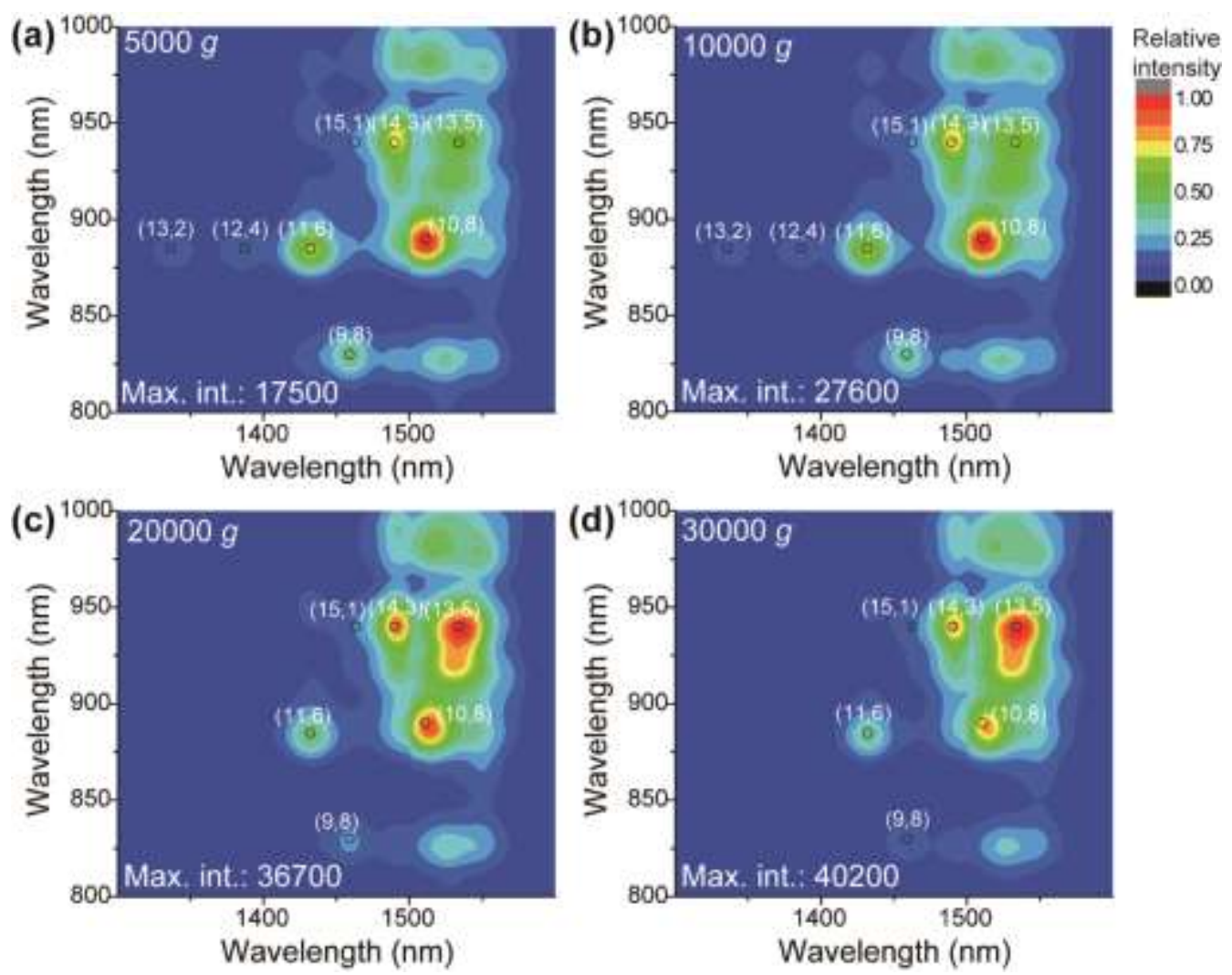

Figure S1. $(\mathrm{a}-\mathrm{d})$ The corresponding PLE maps of FC12-PSWNTs according to varying $g$ forces.
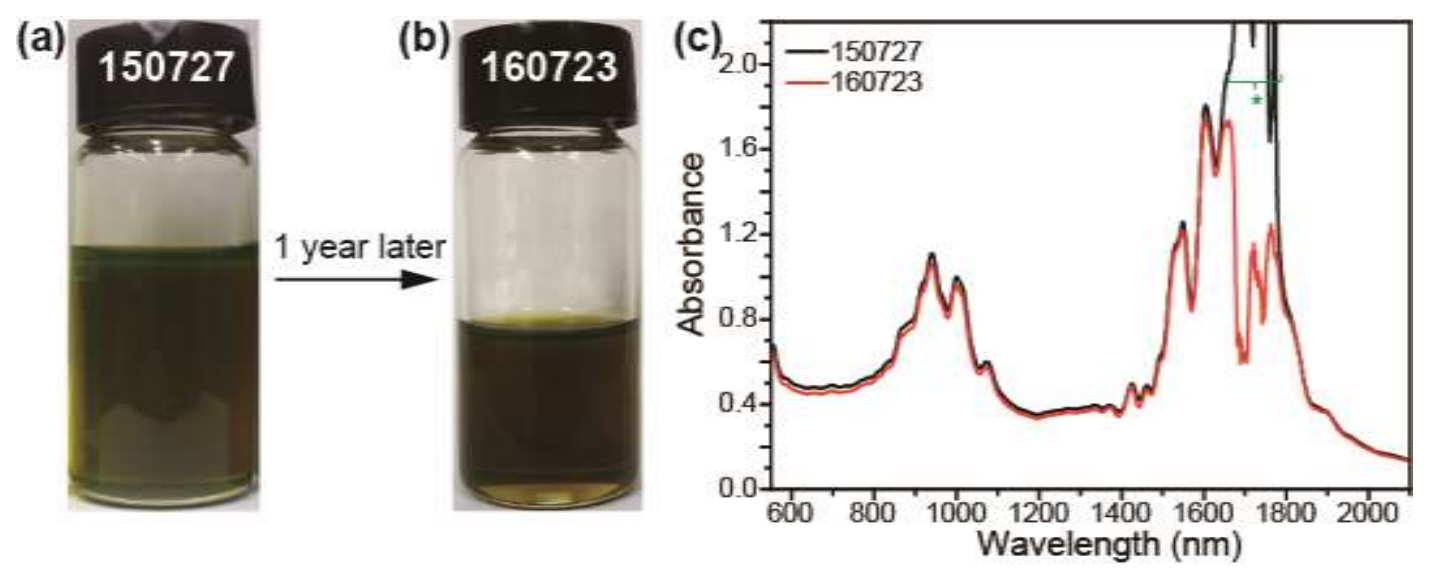

Figure S2. Stability of FC12-PSWNT sample. Photographs of (a) the initial and (b) one year old sample. The reduction in the dispersion is owing to a measurement. (c) The corresponding absorption spectra. An asterisk indicates the absorption attributed to the solvent. 


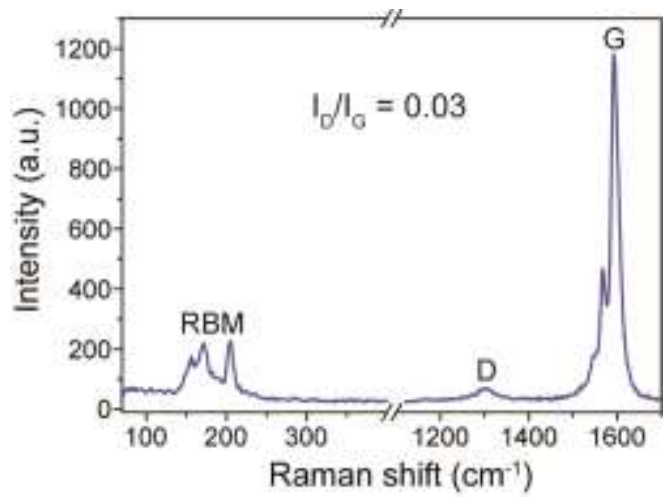

Figure S3. Raman spectra of the as-supplied PSWNT. Laser excitation: $785 \mathrm{~nm}$.

(a)

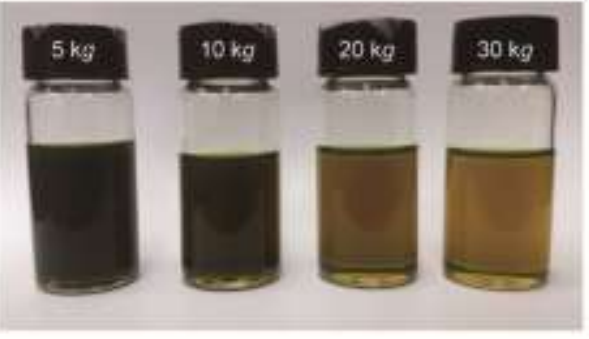

(b)

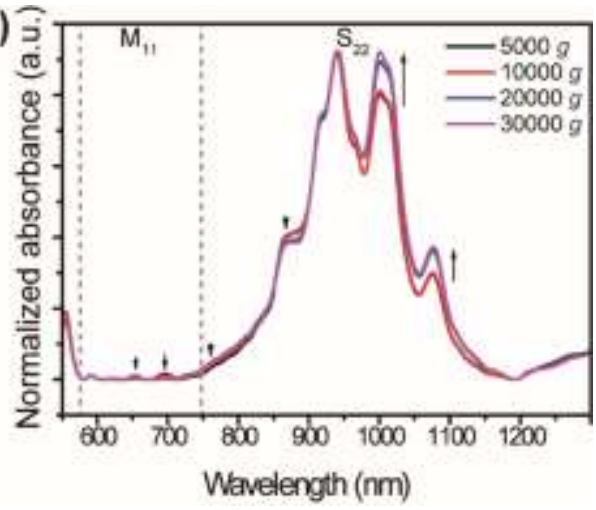

Figure S4. Background-subtracted 940-nm normalized absorption spectra of Figure 3 as a function of centrifugal force. (a) Photograph of FC12-SWNT dispersions obtained from various centrifugal forces. (b) The corresponding normalized absorption spectra based on $940 \mathrm{~nm}$ band.

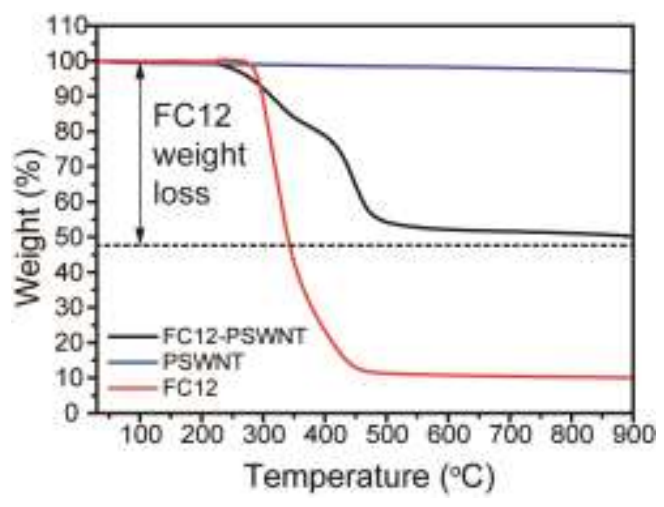

Figure S5. TGA of FC12, PSWNT, and excess FC12 removed, photoreduced FC12-PSWNT. 


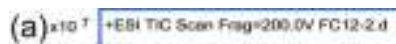

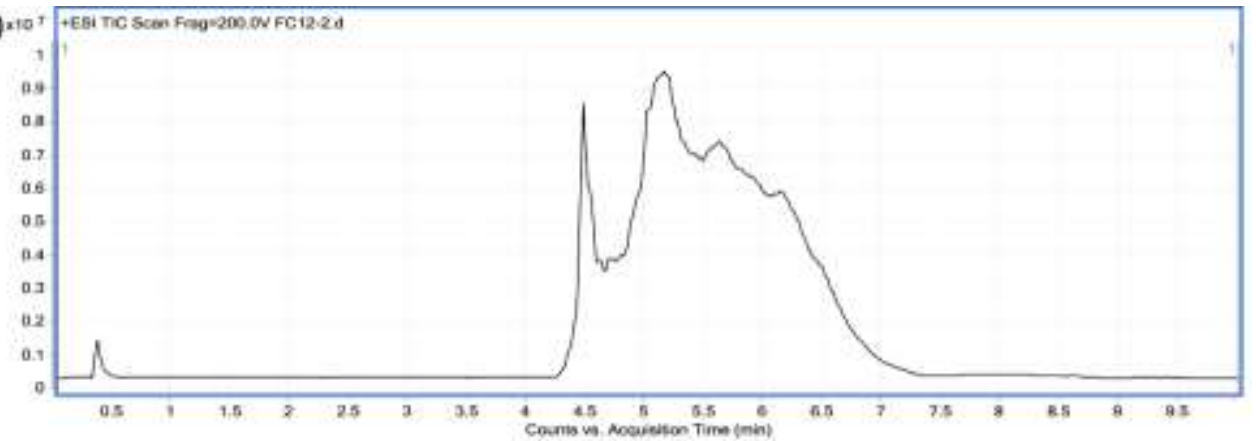

(b)

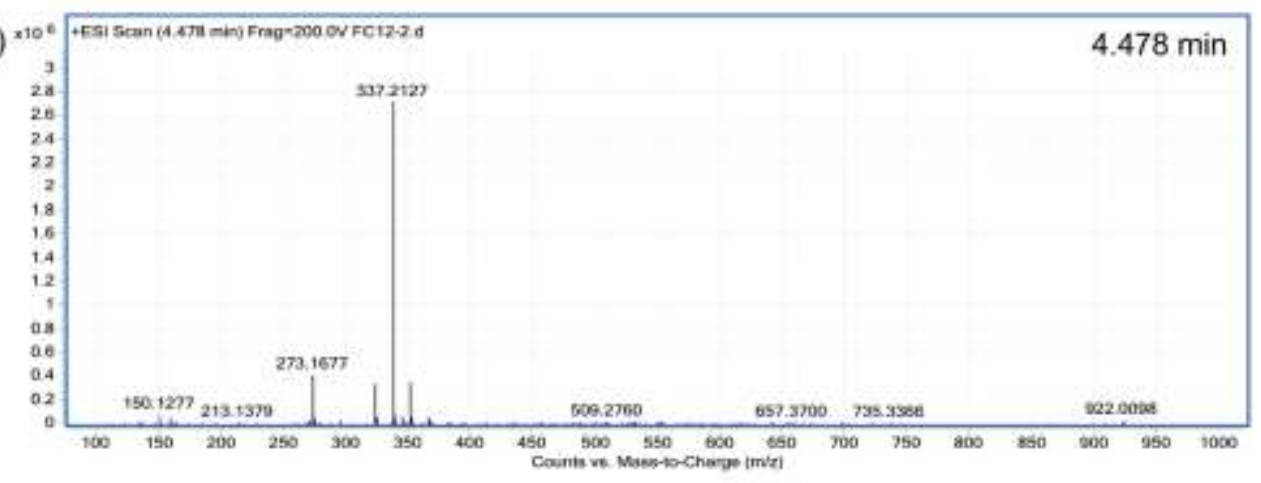

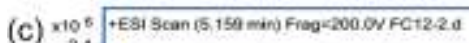

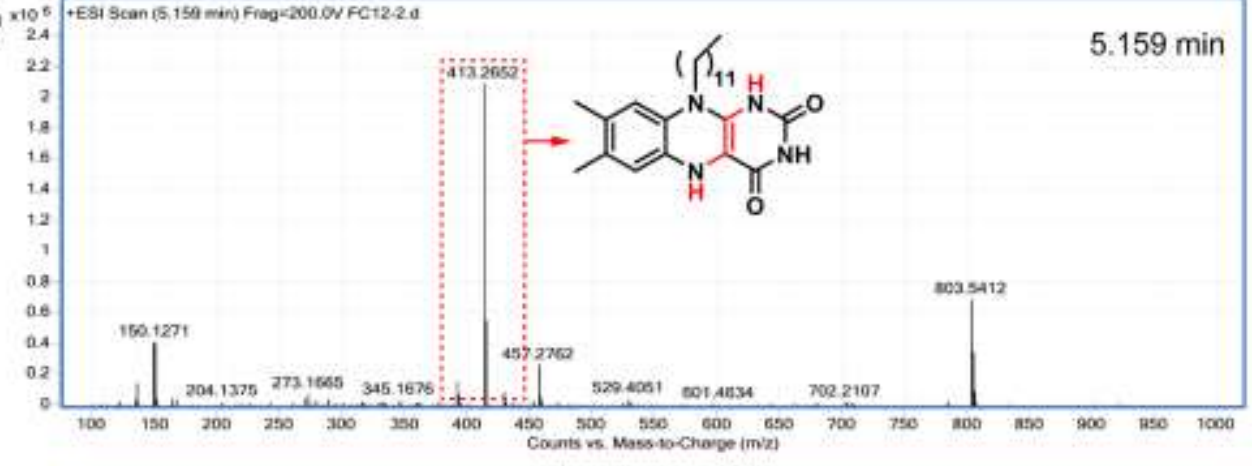

(d)

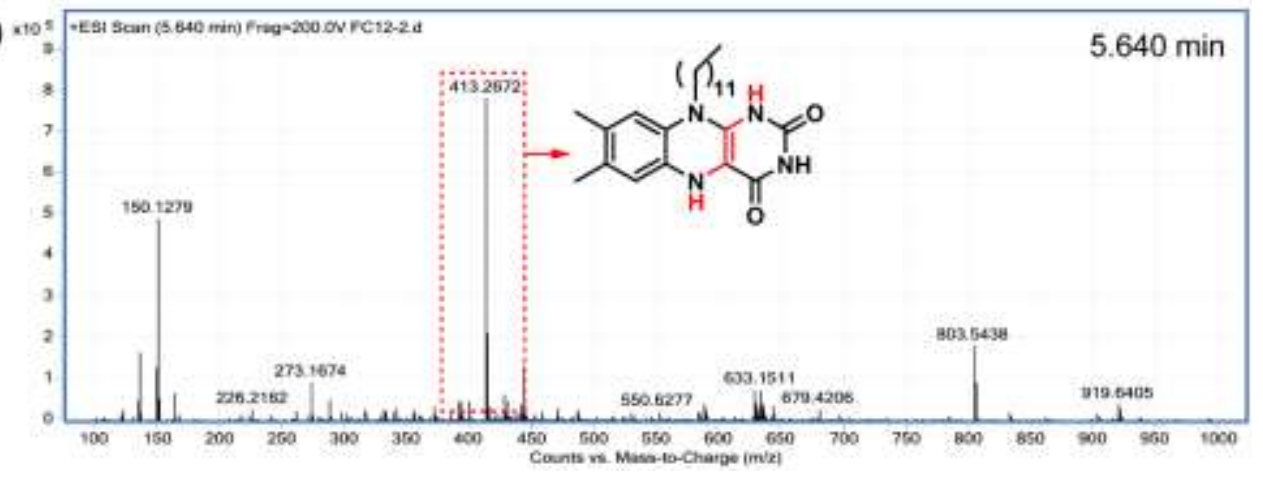

Figure S6. (a) Chromatogram of photoreduced solution, and (b-d) MS spectra from selected acquisition times. 


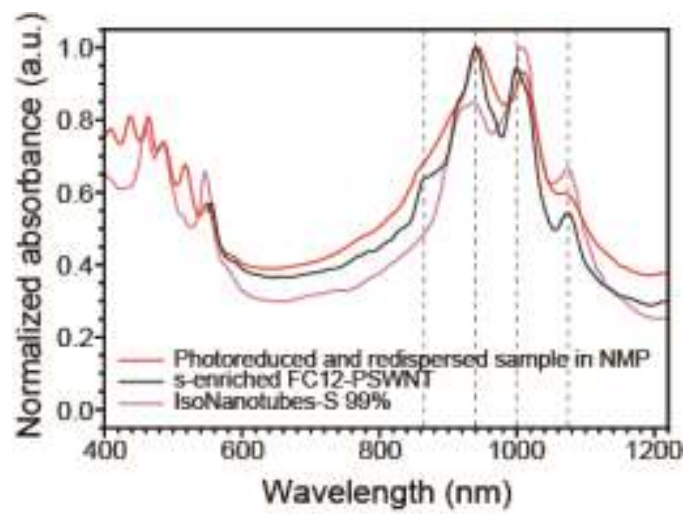

Figure S7. Comparison of absorption spectra of s-enriched FC12-PSWNT (black) and the corresponding photoreduced and redispersed sample in NMP (red), as compared to that of
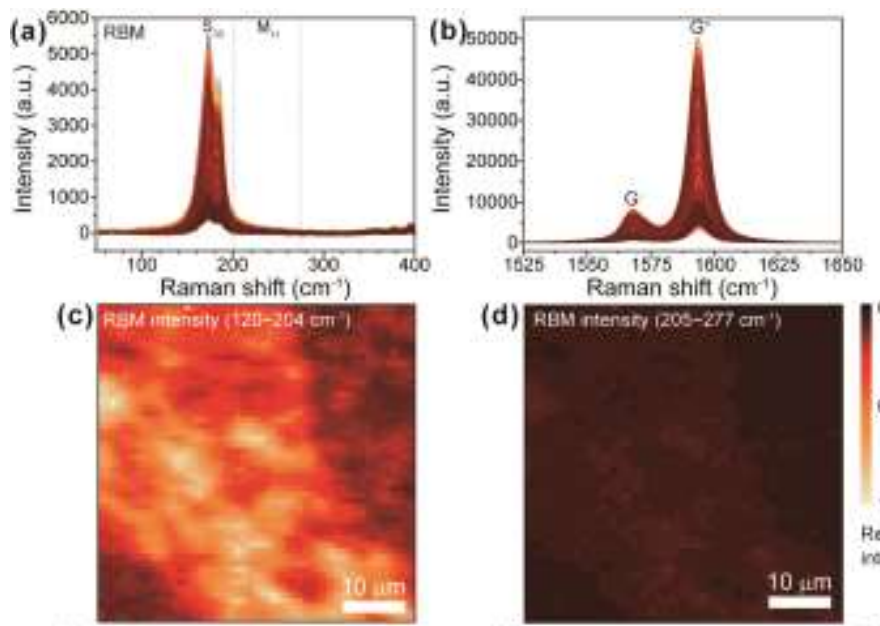

(d) REM ntensay (200-2य) an'?
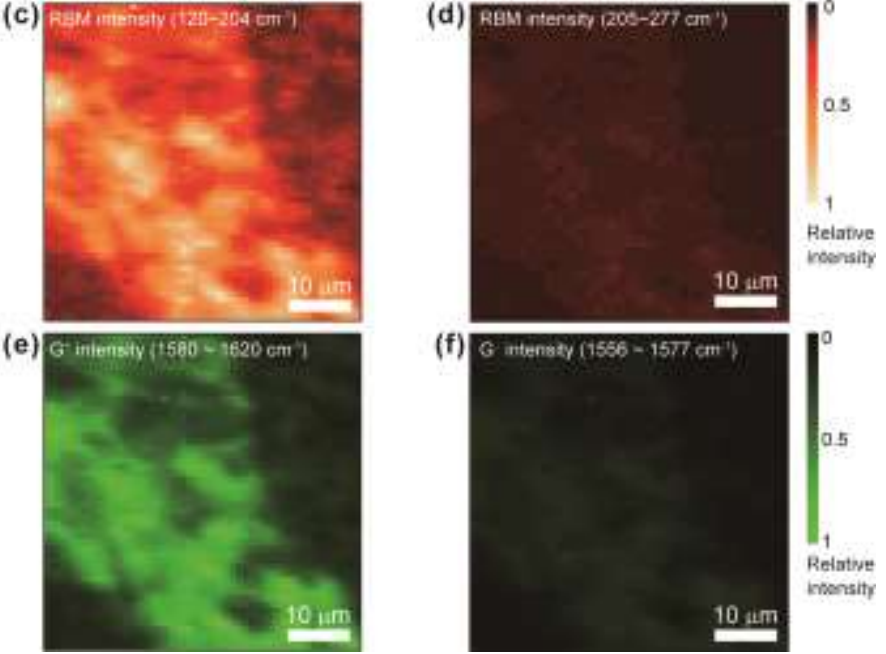

commercial IsoNanotubes-S 99\%.

Figure S8. Raman mapping of s-PSWNT deposited on a Si substrate. (a) RBM and (b) G band spectra from 2500 different points. Summated intensities of RBM regions of (c) $120-204 \mathrm{~cm}^{-1}$ 
from s-PSWNTs and (d) 205-277 $\mathrm{cm}^{-1}$ from m-PSWNTs. Summated intensities of (e) $\mathrm{G}^{+}$(1580$\left.1620 \mathrm{~cm}^{-1}\right)$ and (f) $\mathrm{G}^{-}\left(1556-1577 \mathrm{~cm}^{-1}\right)$ bands. 

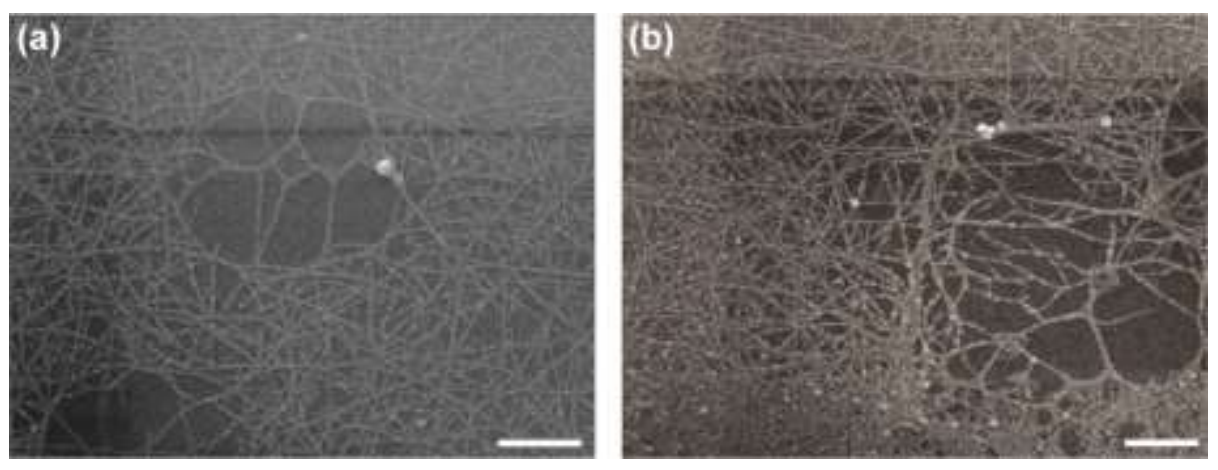

Figure S9. (a - b) SEM images of s-enriched PSWNT deposited on device channel. Scale bar: 1 $\mu \mathrm{m}$.

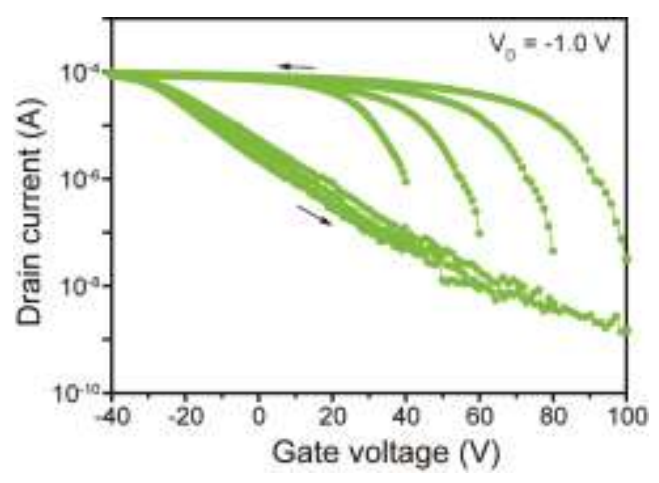

Figure S10. $\mathrm{I}_{D^{-}} \mathrm{V}_{\mathrm{G}}$ transfer curves at different starting $\mathrm{V}_{\mathrm{G}}$ without $\mathrm{Al}_{2} \mathrm{O}_{3}$ deposition.
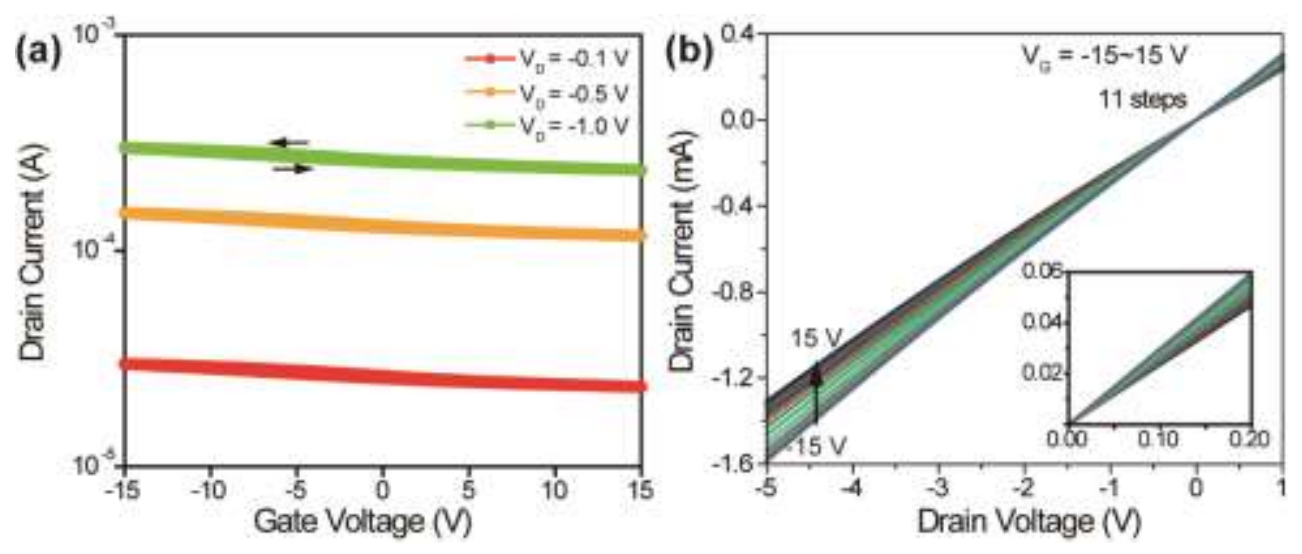

Figure S11. (a) Current-gate voltage transfer and (b) output characteristics of the TFT device prepared by using m-enriched SWNTs. 
Table S1. $\mathrm{S}_{11}$ and $\mathrm{S}_{22}$ positions of FC12- s-SWNTs dispersion in $p$-xylene, based on PLE maps. SWNT diameter was calculated from $1.44 \AA \mathrm{C}-\mathrm{C}$ bond length.

\begin{tabular}{|c|c|c|c|c|c|}
\hline Assignment & Diameter & Optical transitions & \multicolumn{2}{l|}{ Observed $(n, m)$} \\
\cline { 3 - 6 }$(n, m)$ & $(\mathrm{nm})$ & $\mathrm{S}_{11}(\mathrm{~nm})$ & $\mathrm{S}_{22}(\mathrm{~nm})$ & HiPco & PSWNTs \\
\hline$(6,5)$ & 0.76 & 986.7 & 581.9 & $\circ$ & \\
\hline$(8,3)$ & 0.78 & 980.8 & 673.8 & $\circ$ & \\
\hline$(7,5)$ & 0.83 & 1044.9 & 656.9 & $\circ$ & \\
\hline$(8,4)$ & 0.84 & 1124.6 & 605.8 & $\circ$ & \\
\hline$(10,2)$ & 0.88 & 1069.0 & 751.2 & $\circ$ & \\
\hline$(7,6)$ & 0.90 & 1147.4 & 662.0 & $\circ$ & \\
\hline$(9,4)$ & 0.92 & 1118.6 & 736.3 & $\circ$ & \\
\hline$(11,1)$ & 0.92 & 1303.3 & 624.3 & $\circ$ & \\
\hline$(10,3)$ & 0.94 & 1273.9 & 645.4 & $\circ$ & \\
\hline$(8,6)$ & 0.97 & 1205.7 & 734.0 & $\circ$ & \\
\hline$(9,5)$ & 0.98 & 1269.2 & 689.5 & $\circ$ & \\
\hline$(11,3)$ & 1.01 & 1217.6 & 823.4 & $\circ$ & \\
\hline$(13,0)$ & 1.03 & 1418.3 & 687.8 & $\circ$ & \\
\hline$(8,7)$ & 1.03 & 1308.1 & 741.6 & $\circ$ & \\
\hline$(10,5)$ & 1.05 & 1280.9 & 823.8 & $\circ$ & \\
\hline$(11,4)$ & 1.07 & 1413.1 & 729.9 & $\circ$ & \\
\hline$(9,7)$ & 1.10 & 1369.6 & 825.6 & $\circ$ & \\
\hline$(10,6)$ & 1.11 & 1420.1 & 765.8 & $\circ$ & \\
\hline$(13,2)$ & 1.12 & 1334.6 & 883.4 & $\circ$ & $\circ$ \\
\hline$(12,4)$ & 1.15 & 1383.4 & 883.1 & $\circ$ & $\circ$ \\
\hline$(9,8)$ & 1.17 & 1458.7 & 829.4 & $\circ$ & $\circ$ \\
\hline$(11,6)$ & 1.19 & 1432.4 & 884.9 & $\circ$ & $\circ$ \\
\hline$(15,1)$ & 1.23 & 1463 & 940 & $\circ$ & $\circ$ \\
\hline$(10,8)$ & 1.24 & 1512.7 & 889.1 & $\circ$ & $\circ$ \\
\hline$(14,3)$ & 1.25 & 1489.8 & 941.9 & $\circ$ & $\circ$ \\
\hline$(13,5)$ & 1.28 & 1534.6 & 936.6 & $\circ$ & $\circ$ \\
\hline & & & & & \\
\hline
\end{tabular}


Table S2. Absorption-based semiconductor purity and yields of s-PSWNTs from various protocols.

\begin{tabular}{|c|c|c|c|c|c|}
\hline \multicolumn{2}{|c|}{ Sample } & $\begin{array}{c}\text { Peak area ratio of } \\
\mathrm{S}_{22} / \mathrm{M}_{11}\end{array}$ & $\begin{array}{c}\text { Abundance ratio } \\
\text { of s-/m-PSWNT }\end{array}$ & $\begin{array}{c}\text { s-PSWNT } \\
\text { purity (\%) }\end{array}$ & $\begin{array}{c}\text { s-PSWNT } \\
\text { yield (\%) }\end{array}$ \\
\hline \multirow{3}{*}{$\begin{array}{c}\text { FC12- } \\
\text { PSWNT }\end{array}$} & $5000 \mathrm{~g}$ & 250.8 & 57.38 & 98.28 & 13 \\
\cline { 2 - 6 } & $10000 \mathrm{~g}$ & 349.5 & 79.97 & 98.76 & 10.4 \\
\cline { 2 - 6 } & $20000 \mathrm{~g}$ & 1152 & 263.7 & 99.62 & 4.8 \\
\cline { 2 - 6 } & $30000 \mathrm{~g}$ & 1218 & 278.7 & 99.64 & 3.9 \\
\hline \multicolumn{2}{|c|}{ SDS-PSWNT } & 8.741 & 2.000 & 66.67 & \\
\hline
\end{tabular}


Cited references

(S1)Hennrich, F.; Krupke, R.; Lebedkin, S.; Arnold, K.; Fischer, R.; Resasco, D. E.; Kappes, M. M., Raman Spectroscopy of Individual Single-Walled Carbon Nanotubes from Various Sources. J. Phys. Chem. B 2005, 109 (21), 10567-10573.

(S2)Bachilo, S. M.; Strano, M. S.; Kittrell, C.; Hauge, R. H.; Smalley, R. E.; Weisman, R. B., Structure-Assigned Optical Spectra of Single-Walled Carbon Nanotubes. Science 2002, 298 (5602), 2361-2366.

(S3)Nikolaev, P.; Bronikowski, M. J.; Bradley, R. K.; Rohmund, F.; Colbert, D. T.; Smith, K. A.; Smalley, R. E., Gas-Phase Catalytic Growth of Single-Walled Carbon Nanotubes from Carbon Monoxide. Chem. Phys. Lett. 1999, 313 (1-2), 91-97.

(S4)Itkis, M. E.; Perea, D. E.; Jung, R.; Niyogi, S.; Haddon, R. C., Comparison of Analytical Techniques for Purity Evaluation of Single-Walled Carbon Nanotubes. J. Phys. Soc. Japan 2005, 127 (10), 3439-3448.

(S5)Tanaka, T.; Jin, H.; Miyata, Y.; Fujii, S.; Suga, H.; Naitoh, Y.; Minari, T.; Miyadera, T.;

Tsukagoshi, K.; Kataura, H., Simple and Scalable Gel-Based Separation of Metallic and Semiconducting Carbon Nanotubes. Nano Lett. 2009, 9 (4), 1497-1500.

(S6)Antaris, A. L.; Seo, J.-W. T.; Green, A. A.; Hersam, M. C., Sorting Single-Walled Carbon Nanotubes by Electronic Type Using Nonionic, Biocompatible Block Copolymers. ACS Nano 2010, 4 (8), 4725-4732.

(S7)Sundramoorthy, A. K.; Mesgari, S.; Wang, J.; Kumar, R.; Sk, M. A.; Yeap, S. H.; Zhang, Q.; Sze, S. K.; Lim, K. H.; Chan-Park, M. B., Scalable and Effective Enrichment of Semiconducting Single-Walled Carbon Nanotubes by a Dual Selective Naphthalene-Based Azo Dispersant. J. Am. Chem. Soc. 2013, 135 (15), 5569-5581.

(S8)Mistry, K. S.; Larsen, B. A.; Blackburn, J. L., High-Yield Dispersions of Large-Diameter Semiconducting Single-Walled Carbon Nanotubes with Tunable Narrow Chirality Distributions. ACS Nano 2013, 7 (3), 2231-2239.

(S9)Finnie, P.; Ding, J.; Li, Z.; Kingston, C. T., Assessment of the Metallicity of Single-Wall Carbon Nanotube Ensembles at High Purities. J. Phys. Chem. C 2014, 118 (51), 3012730138. 